\title{
Contribuições da Modelagem Matemática em Meios Porosos para o Ensino de Funções
}

\author{
Paula R. L. Couto, \\ Depto de Matemática, UFPR, \\ 81531-980, Curitiba, PR \\ E-mail: paulacouto@ufpr.br \\ Sandra M. C. Malta \\ LNCC - Coordenação de Matemática Aplicada \\ 88040-900, Petrópolis, RJ \\ E-mail: smcm@lncc.br.
}

\begin{abstract}
Resumo: Este artigo versa sobre a obtenção de três modelos matemáticos para a fração de volume de um meio poroso bifásico. Os objetivos são explicitar como este processo promove a interação de vários campos da matemática básica e exemplificar como surgem as expressões finais de algumas funções que são estudadas no Ensino Médio e Superior. Assuntos como continuidade e derivadas também são abordados para investigar algumas potencialidades dos modelos obtidos. Concluímos que a modelagem matemática é um ferramenta dinâmica e interativa para o processo de ensino-aprendizagem de funções.
\end{abstract}

Palavras-chave: Fração de volume, Meios porosos, Funções, Modelagem Matemática.

\section{Introdução}

Neste artigo exibimos a dedução dos modelos da função fração de volume, apresentados em [4] e inseridos na ampla problemática da modelagem de escoamento em meios porosos. A proposta é exemplificar, através desta modelagem, como surgem as expressões finais de algumas funções que são estudadas no Ensino Médio e Superior e que são abordadas, de modo geral, a partir da análise de sua fórmula pronta. Além disto, desejamos explicitar como este processo promove a interação de vários campos da matemática básica. Baseados no entendimento de que criticar e alterar modelos matemáticos existentes, além de contruí-los, são habilidades desejáveis em prováveis usuários de modelos matemáticos [5], pretendemos contribuir com o aumento do número de referências sobre modelagem matemática com reais possibilidades de aplicação nas salas de aula do Ensino Médio ou Superior, em cursos de formação de professores de matemática, ou ainda em programas de iniciação científica.

Partimos primeiramente da introdução do significado da fração de volume dentro do contexto de sistemas bifásicos em meios porosos. Em seguida introduzimos um modelo conceitual simples de um meio poroso no qual a fração de volume poderá ser entendida como uma função real de uma variável real, definimos também três formas geométricas que são assumidas como possíveis volumes de controle e, na sequência, apresentamos a modelagem de uma delas (devido à restrição de espaço neste artigo) e as demais são apenas apresentadas (a dedução é feita de forma análoga). Finalmente, discutimos as contribuições da modelagem apresentada para o estudo de funções e para a Educação Matemática e exibimos nossas considerações finais. 


\section{A fração de volume de um meio poroso}

Meios porosos aparecem em problemas de várias áreas, como nas engenharias química, ambiental, civil e do petróleo, dentre outras. Podemos citar vários exemplos de meios porosos naturais que são alvos conhecidos de interesses, tais como reservatórios de petróleo e de águas subterrâneas. Um meio poroso pode ser definido, de forma simplificada, com uma matriz sólida com vazios dentro, os poros, os quais estão normalmente preenchidos por um fluido como ar, água ou petróleo ou por vários fluidos, misturados ou separados por um contorno bem definido. Cada porção do sistema (meio poroso) que é separada das outras por um contorno bem definido é chamada de fase, por exemplo, a matriz sólida pode constituir uma das fases, a fase sólida, e os vazios podem estar preenchidos por várias fases como por exemplo uma fase líquida e outra gasosa (água e ar ou petróleo e gás natural) [3]. Admitimos aqui um meio poroso bifásico constituído por apenas duas fases : a fase sólida (fase $\alpha$ ) e um fase que preenche os poros (fase $\beta$ ). Além disso, supomos que o meio poroso possui uma estrutura de placas paralelas com seção uniforme ao longo do eixo $z$ como a mostrada na Figura 1, onde a parte hachurada corresponde à matriz sólida.

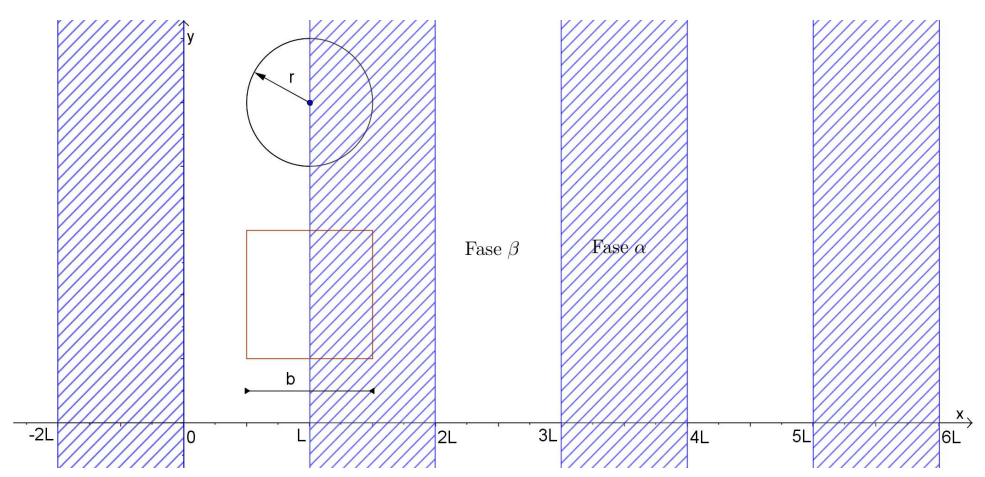

Figura 1: Seção do meio poroso considerado. O círculo representa a seção de um volume de controle esférico ou um cilindro circular reto e o retângulo a seção de um paralelepípedo. Figura adaptada de [4].

A fração de volume que aparece no contexto da modelagem macroscópica de escoamentos de fluidos em meios porosos é a razão entre o volume ocupado por uma das fases e o volume de controle $V$. No caso do meio poroso bifásico temos as seguintes relações: $V=V_{\alpha}+V_{\beta}$ onde $V_{\alpha}$ é o volume ocupado pela fase $\alpha$ no volume de controle e $V_{\beta}$ é o volume ocupado pela fase $\beta$ no volume de controle. Ao dividirmos esta relação por $V$ obtemos $1=V_{\alpha} / V+V_{\beta} / V=\epsilon_{\alpha}+\epsilon_{\beta}$, onde $\epsilon_{\alpha}=V_{\alpha} / V$ é a fração de volume da fase $\alpha$ e $\epsilon_{\beta}=V_{\beta} / V$ é a fração de volume da fase $\beta$. Para fins de aplicações, a fração de volume mais interessante é a última $\left(\epsilon_{\beta}\right)$, pois ela está relacionada com a medida da capacidade do meio poroso de permitir que um fluido escoe dentro dele [3] e é ela que vamos modelar para três volumes de controle distintos: um paralelepípedo, um cinlindro circular reto e uma esfera (cujas seções estão representadas na Figura 1).

Quando um cilindro circular reto é tomado como o volume de controle, a razão entre $V_{\beta}$ e $V$, isto é, $\epsilon_{\beta}$, depende tanto do raio do cilindro circular reto, quanto da posição em que ele se encontra. Vamos definir sua posição a partir do centro do círculo, que será chamado de centróide, e fixamos um valor para o raio de $r=L / 2$. Para determinarmos quais são as possíveis razões entre $V_{\beta}$ e $V$ podemos imaginar o centróide como um ponto móvel que se movimenta na direção do semi-eixo positivo $x$ e, desta forma, as possíveis configurações para os volumes podem ser observadas (Figuras 2 e 3 ).

Assim, quando o centróide está na posição $x=0$, o volume ocupado pela fase $\beta$ é a metade do volume de controle, portanto $\epsilon_{\beta}(0)=V_{\beta} / V=0.5 V / V=1 / 2$. À medida que o centróide assume posições $0<x<L / 2$, o volume ocupado pela fase $\beta$ se torna maior que o volume ocupado pela fase $\alpha$ (no caso em que $r=L / 2$ ) e assume a configuração mais à esquerda da Figura 4. Neste caso, o volume da fase $\beta$ é dado por $V_{\beta}=V-V_{\alpha}$, onde $V_{\alpha}$ é o volume da fase 

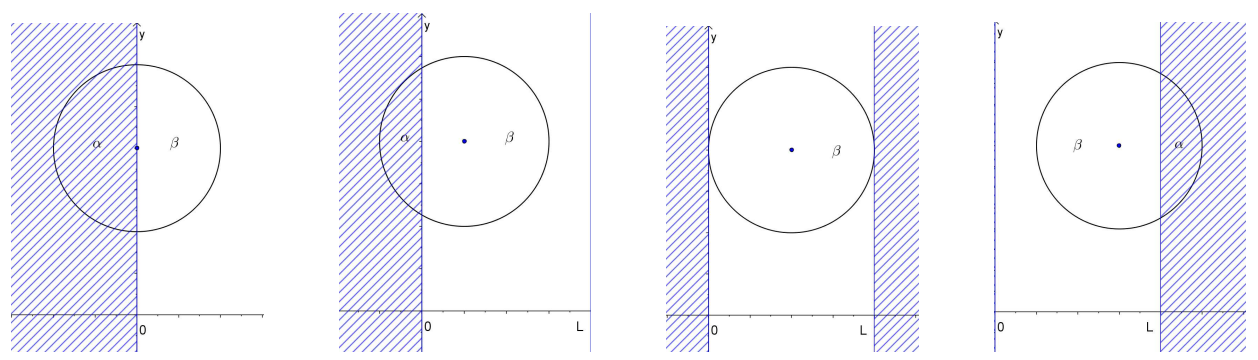

Figura 2: Configurações possíveis para a relação entre $V_{\beta}$ e $V$ com a posição $x$ do centróide variando da esquerda para a direita para $0 \leq x<L$.
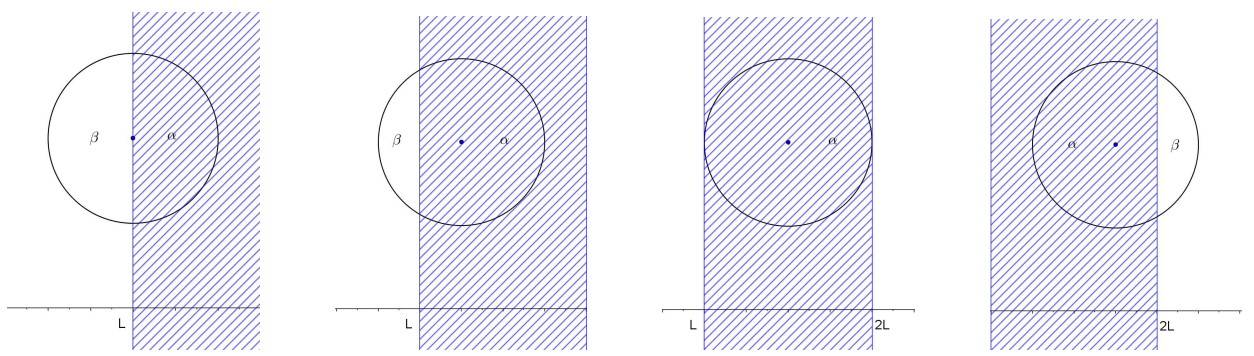

Figura 3: Configurações possíveis para a relação entre $V_{\beta}$ e $V$ com a posição $x$ do centróide variando da esquerda para a direita para $L \leq x<2 L$.

$\alpha$. Para calcular o volume ocupado pela fase $\alpha$, calculamos a área da região hachurada $\left(A_{\alpha}\right)$ subtraindo a área do triângulo $A C B(\triangle A C B)$ da área do setor circular $A C B$. Sendo $D C=x$ e $D A=\sqrt{r^{2}-x^{2}}$ temos:

$$
\begin{gathered}
\text { área do triângulo } A C B=2 \text { área do triângulo } A D C=x \sqrt{r^{2}-x^{2}}, \\
\text { área do setor } A C B=r^{2} \frac{\theta}{2}=r^{2} \operatorname{sen}^{-1}\left(\frac{\sqrt{r^{2}-x^{2}}}{r}\right), \\
A_{\alpha}=r^{2} \operatorname{sen}^{-1}\left(\frac{\sqrt{r^{2}-x^{2}}}{r}\right)-x \sqrt{r^{2}-x^{2}} .
\end{gathered}
$$

Desta forma,

$$
A_{\beta}=\pi r^{2}+x \sqrt{r^{2}-x^{2}}-r^{2} \operatorname{sen}^{-1}\left(\frac{\sqrt{r^{2}-x^{2}}}{r}\right),
$$

onde $A_{\beta}$ é a área relativa à fase $\beta$.

Logo,

$$
\epsilon_{\beta}(x)=\frac{V_{\beta}}{V}=\frac{\pi r^{2}+x \sqrt{r^{2}-x^{2}}-r^{2} \operatorname{sen}^{-1}\left(\frac{\sqrt{r^{2}-x^{2}}}{r}\right)}{\pi r^{2}}
$$

e sendo $r=L / 2$ temos

$$
\epsilon_{\beta}(x)=1+\frac{2 x}{\pi L^{2}} \sqrt{L^{2}-4 x^{2}}-\frac{1}{\pi} \operatorname{sen}^{-1}\left(\frac{\sqrt{L^{2}-4 x^{2}}}{L}\right) .
$$

Quando $x=L / 2$, o volume de controle contém apenas a fase $\beta$. Portanto $\epsilon_{\beta}(L / 2)=V_{\beta} / V=$ $V / V=1$. Quando $L / 2<x<L$ o volume ocupado pela fase $\beta$ começa a diminuir a medida que $x$ 

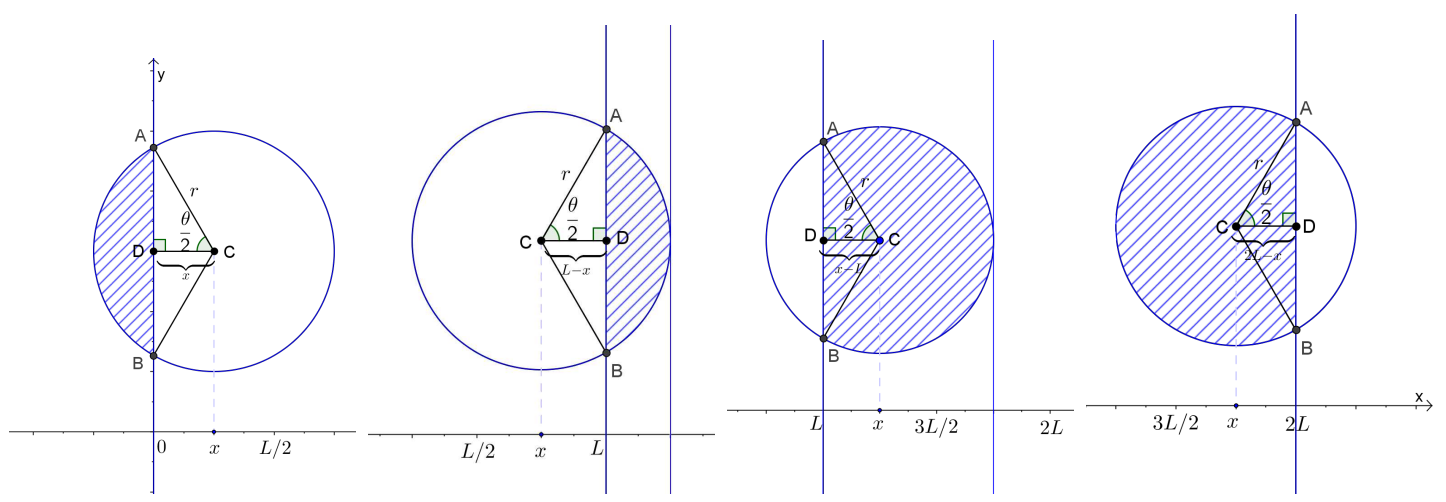

Figura 4: Da esquerda para direita as configurações das relações entre $V_{\beta}$ e $V$ nos casos em que $0<x<L / 2, L / 2<x<2 L, L<x<3 L / 2$ e $L / 2<x<2 L$, respectivamente.

avança para a direita (segunda configuração da esquerda para a direita na Figura 4). Novamente temos $A_{\alpha}=$ área do setor $A C B$ - área do triângulo $A C B=\theta r^{2} / 2-(L-x) \sqrt{r^{2}-(L-x)^{2}}$. Como $\theta / 2=\pi / 2-\operatorname{sen}^{-1}((L-x) / r)$ temos

$$
A_{\alpha}=\frac{\pi r^{2}}{2}-r^{2} \operatorname{sen}^{-1}\left(\frac{L-x}{r}\right)-(L-x) \sqrt{r^{2}-(L-x)^{2}} .
$$

Sendo $r=L / 2$ chegamos a

$$
A_{\beta}=\frac{\pi L^{2}}{4}-A_{\alpha}=\frac{\pi L^{2}}{8}+\frac{L^{2}}{4} \operatorname{sen}^{-1}\left(\frac{2(L-x)}{L}\right)+\frac{(L-x)}{2} \sqrt{L^{2}-4(L-x)^{2}} .
$$

Logo, para $L / 2<x<L$

$$
\epsilon_{\beta}(x)=\frac{V_{\beta}}{V}=\frac{A_{\beta}}{\pi L^{2} / 4}=\frac{1}{2}+\frac{2(L-x)}{\pi L^{2}} \sqrt{L^{2}-4(L-x)^{2}}+\frac{1}{\pi} \operatorname{sen}^{-1}\left(\frac{2(L-x)}{L}\right) .
$$

Quando $x=L, \epsilon_{\beta}(x)=V_{\beta} / V=(V / 2) / V=1 / 2$. ̀̀ medida que $x$ avança para a direita assumindo valores $L<x<3 L / 2$ (terceira configuração da esquerda para a direita na Figura 4) o volume ocupado pela fase $\beta$ continua a diminuir até $x=3 L / 2$ onde $V_{\beta}=0$. Neste intervalo temos $A_{\beta}=$ área do setor $A C B$ - área do triângulo $A C B$, isto é,

$$
A_{\beta}=\left(\frac{\pi}{2}-\operatorname{sen}^{-1}\left(\frac{x-L}{r}\right)\right) r^{2}-(x-L) \sqrt{r^{2}-(x-L)^{2}} .
$$

Para $r=L / 2$ obtemos

$$
A_{\beta}=\frac{\pi L^{2}}{8}-\operatorname{sen}^{-1}\left(\frac{2(x-L)}{L}\right) \frac{L^{2}}{4}-\frac{(x-L)}{2} \sqrt{L^{2}-4(x-L)^{2}} .
$$

Como a função $y=\operatorname{sen}^{-1}(x)$ é ímpar, podemos escrever $-\operatorname{sen}^{-1}((2(x-L)) / L)=\operatorname{sen}^{-1}((2(L-$ $x)) / L)$. Além disso, escrevendo $x-L=-(L-x)$ em $A_{\beta}$ acima obtemos

$$
\epsilon_{\beta}(x)=\frac{1}{2}+\frac{1}{\pi} \operatorname{sen}^{-1}\left(\frac{2(L-x)}{L}\right)+\frac{2(L-x)}{\pi L^{2}} \sqrt{L^{2}-4(x-L)^{2}} .
$$

A partir de $x=3 L / 2$ o valor de $V_{\beta}$ começa a crescer novamente (última configuração da esquerda para a direita na Figura 4) até chegar em $x=2 L$. Neste intervalo, $3 L / 2<x<2 L$, temos $A_{\beta}=$ área do setor $A C B$ - área do triângulo $A C B$, isto é, 


$$
A_{\beta}=\left(\frac{\pi}{2}-\operatorname{sen}^{-1}\left(\frac{2 L-x}{r}\right)\right) r^{2}-(2 L-x) \sqrt{r^{2}-(2 L-x)^{2}} .
$$

Para $r=L / 2$ temos

$$
A_{\beta}=\frac{\pi L^{2}}{8}-\frac{L^{2}}{4} \operatorname{sen}^{-1}\left(\frac{2(2 L-x)}{L}\right)-\frac{(2 L-x)}{2} \sqrt{L^{2}-4(2 L-x)^{2}} .
$$

Portanto,

$$
\epsilon_{\beta}(x)=\frac{1}{2}-\frac{1}{\pi} \operatorname{sen}^{-1}\left(\frac{2(2 L-x)}{L}\right)-\frac{2(2 L-x)}{\pi L^{2}} \sqrt{L^{2}-4(2 L-x)^{2}} .
$$

Quando $x=2 L$ atinge-se novamente a configuração inicial e o comportamento descrito vai se repetindo a cada $2 L$, ou seja, a função $\epsilon_{\beta}$ é periódica com período $2 L$.

Portanto, a função que representa a fração de volume do meio poroso em estudo, quando usamos um volume de controle cilíndrico, no intervalo $[0,2 L]$ é uma expressão definida por partes dada por

$$
\epsilon_{\beta}(x)=\left\{\begin{array}{l}
1+\frac{2 x}{\pi L^{2}} \sqrt{L^{2}-4 x^{2}}-\frac{1}{\pi} \operatorname{sen}^{-1}\left(\frac{\sqrt{L^{2}-4 x^{2}}}{L}\right), \quad \text { se } \quad 0 \leq x<L / 2, \\
\frac{1}{2}+\frac{1}{\pi} \operatorname{sen}^{-1}\left(\frac{2(L-x)}{L}\right)+\frac{2(L-x)}{\pi L^{2}} \sqrt{L^{2}-4(x-L)^{2}}, \quad \text { se } \quad L / 2 \leq x<3 L / 2, \\
\frac{1}{2}-\frac{1}{\pi} \operatorname{sen}^{-1}\left(\frac{2(2 L-x)}{L}\right)-\frac{2(2 L-x)}{\pi L^{2}} \sqrt{L^{2}-4(2 L-x)^{2}}, \quad \text { se } \quad 3 L / 2 \leq x<2 L .
\end{array}\right.
$$

Com um raciocínio análogo, usando um paralelepípedo com largura $b=L$ e uma esfera de raio $L / 2$ como volumes de controle, chegamos aos seguintes modelos, respectivamente:

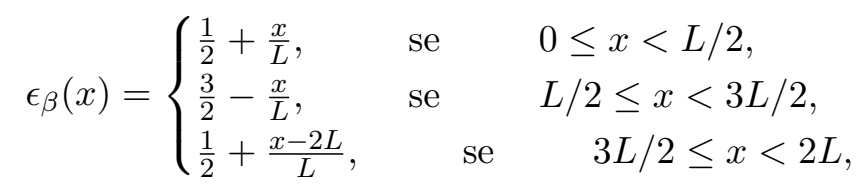

e

$$
\epsilon_{\beta}(x)=\left\{\begin{array}{llrl}
\frac{1}{2}+\frac{3 x}{2 L^{3}}\left[L^{2}-\frac{4 x^{2}}{3}\right], & \text { se } & \multicolumn{2}{c}{0 \leq x / 2,} \\
\frac{1}{2}+\frac{3(L-x)}{2 L^{3}}\left[L^{2}-\frac{4(L-x)^{2}}{3}\right], & \text { se } & L / 2 \leq x<3 L / 2, \\
\frac{1}{2}+\frac{3(x-2 L)}{2 L^{3}}\left[L^{2}-\frac{4(x-2 L)^{2}}{3}\right], & \text { se } & 3 L / 2 \leq x<2 L .
\end{array}\right.
$$

\section{Resultados e Discussões}

Podemos observar que as Equações (1), (2) e (3) representam funções que são definidas por partes, as quais envolvem funções polinomiais, a função raíz quadrada, a função inversa da função seno e também funções compostas. Várias propriedades de funções foram utilizadas na modelagem, tais como a paridade da função inversa da função seno, a periodicidade da função resultante. Desta maneira, os modelos obtidos poderiam fazer parte dos diversos exemplos que temos à disposição nos livros de matemática a respeito do assunto funções. No entanto, o que as destacam dentro deste espectro é o fato de conhecermos a sua história, de sabermos como elas foram obtidas a partir de um problema físico, o problema de modelagem do escoamento em meios poroso, um assunto que é de amplo interesse científico.

Devemos notar que, de acordo com [2], os modelos obtidos poderiam ser classificados como estáticos, no entanto o processo que descrevemos para capturar os seus comportamentos passou pela visualização do centróide como um ponto móvel, o qual nos conduziu à percepção das diferentes configurações necessárias para obter os modelos para a fração de volume.

Estes modelos também merecem destaque, pois são construídos a partir de uma matemática básica, que envolve conceitos de trigonometria (no caso da dedução apresentada), geometria 
espacial (as percepções dos volumes envolvidos em cada caso: volume do cilindro, do paralelepípedo e do volume de esfera e de suas seções), representação cartesiana de pontos, dentre outros tópicos. Esta modelagem poderia ser abordada tanto no Ensino Médio quanto no Ensino Superior.

Perguntas sobre qual dos modelos obtidos melhor representaria a fração de volume de um meio poroso certamente surgiriam num ambiente de discussão sobre modelagem e algumas respostas poderiam ser obtidas com análises sobre a continuidade da função. Investigações sobre a existência de derivadas para cada uma das funções poderiam ser realizadas e levariam a conclusão de que o volume esférico conduz à uma fração de volume com derivadas de todas as ordens em todos os pontos do seu domínio [4] e neste sentido, aconteceria um refinamento ou uma melhora no modelo matemático a partir da mudança da forma do volume de controle empregado. Além disso, questões sobre como seriam os modelos para dimensões maiores dos volumes de controle estudados (aqui assumimos $r=L / 2$ e $b=L$ ) e qual o significado disso para a modelagem de meios porosos também seriam temas de estudo. Perguntas sobre o que aconteceria com o modelo se a geometria do meio poroso fosse diferente daquela do modelo conceitual de placas paralelas apresentado na Figura 1 seriam interessantes temas de pesquisa.

Todas as discussões geradas à partir do processo de modelagem podem passar despercebidas ao se olhar para uma função apenas à partir de sua fórmula pronta. Neste sentido, concordamos com o argumento de [1] de que "É necessário que os alunos tenham a oportunidade de discutir (e experimentar quando for o caso) as circunstâncias que conduziram o pensamento humano para tal representação matemática", não apenas dentro do discurso pedagógico das ciências como química, física e biologia, mas também no discurso pedagógigo da própria matemática.

\section{Considerações Finais}

Neste trabalho, apresentamos a modelagem da fração de volume de um meio poroso com a finalidade de revelar como diferentes estruturas geométricas do volume de controle podem gerar diferentes modelos e como eles podem ser obtidos a partir de uma matemática básica. Além disso, apontamos várias perpectivas de possíveis trabalhos com modelagem matemática a partir deste e também várias de suas contribuições para o estudo de funções e para a Educação Matemática de modo geral. Portanto, concluímos que a modelagem é um caminho dinâmico e interativo para o processo de ensino-aprendizagem de matemática ao apresentar a construção de modelos a partir de um problema físico real cujas expressões são constituídas por funções usuais no Ensino Médio e Superior.

\section{Referências}

[1] J. C. Barbosa, Modelagem e Modelos Matemáticos na Educação Científica, Alexandria (UFSC), 2 (2009) 65-85.

[2] R. C. Bassanezi, "Ensino-aprendizagem com modelagem matemática : uma nova estratégia", Contexto, São Paulo, 2002.

[3] P. R. L. Couto, "Modelagem computacional do transporte de contaminantes com processos de biodegradação e sorção física em um meio poroso saturado ", Tese de Doutorado, LNCC, 2006 .

[4] F. A. Howes, S. Whitaker, The spacial avaraging theorem revisited, Chemical Engineering Science, 40 (1985) 1387-1392.

[5] M. Mesterton-Gibbons, "A concrete approach to mathematical modelling", Willey, NewYork, 1995. 